\title{
A foveal discriminability difference for one vs. four letters
}

\author{
BRUCE A. AMBLER, RAYMOND KEEL, and ELAINE PHELPS \\ University of Texas at Arlington, Arlington, Texas 76019
}

\begin{abstract}
In two experiments using foveal displays, an increase in reaction time was found for a T-L discrimination when number of letters was increased from one to four. No such increase was observed for a T-tilted T discrimination. In Experiment 1, acuity (manipulation of line width) did not interact with the effect of letter number. In Experiment 2, the retinal area (a manipulation of letter size and spacing) covered by the display also failed to affect the influence of letter number. The results indicate that the type of discrimination to be made determines whether or not processing is parallel, even when as few as four letters are presented.
\end{abstract}

Recently, it has been proposed that processing of information presented for letter discrimination in foveal vision is not influenced by letter number. Shiffrin, McKay, and Shaffer (1976) found that accuracy of letter recognition did not vary when subjects were allowed to choose from either one or nine foveal targets. However, this experiment presented methodological problems (Keren \& Shelton, 1976; Shiffrin, 1977). Shiffrin and Gardner (1972) found that accuracy of letter reports was actually worse when subjects attended to one as compared to four letter positions at a time. Recently, however, Prinzmetal and Banks (Note 1) showed that this result depends on the order of presentation when subjects are attending to only one letter at a time. In the one-letter conditions the four letters were presented one at a time with a $100-\mathrm{msec}$ delay between letters. When the spatial order of letter presentation was random, accuracy was higher in the one-letter than in the four-letter condition. The authors suggest that fixed-order presentation encourages eye movements which degrade performance. Ambler and Finklea (1976) found that increasing foveal letter number from one to four letters resulted in a small increase in error rate for a T-L discrimination. However, this change was not significant. As letter number increased to 18 , a significant increase in errors was observed.

Beck and Ambler (1973) showed that T-L discrimination performance was reduced in peripheral vision when number of attended letter positions was increased from one to two. It is possible the number of letters that can be processed simultaneously varies with retinal position. In the fovea there may be enough capacity to process four letters simultaneously, even for a T-L discrimination which clearly required serial processing in the periphery.

A major problem with the acceptance of parallel

Requests for reprints should be sent to Bruce A. Ambler, Department of Psychology, University of Texas at Arlington, Arlington, Texas 76019. processing (processing unaffected by letter number) is that support for it has required acceptance of the null hypothesis. Ambler and Finklea (1976) found no significant differences between one and four letters. However, there were more errors in the four-letter than in the oneletter condition. Would a more sensitive experiment demonstrate a reliable difference between these conditions?

In the following experiments we used a more sensitive reaction time paradigm to investigate the effect of letter number on foveal processing. Letter size and acuity were also varied to see if they would interact with the effect of distributed attention. In addition to the $\mathrm{T}-\mathrm{L}$ discrimination, a T-tilted $\mathrm{T}$ discrimination which had previously been little influenced by letter number (Amber \& Finklea, 1976) was also included.

\section{EXPERIMENT 1}

Previously, differences between one and four letters had been found in the periphery but not in the fovea (Ambler \& Finklea, 1976). In those experiments an attempt was made to control for acuity by making the peripheral letters larger. However, the width of the lines composing the letters was not varied and it is possible that acuity was higher for the foveal letters. Perhaps selective attention is more important under conditions of lower acuity.

In this experiment either 1 or 4 letters was presented foveally for a T-L or a T-tilted T discrimination. The line widths of the letters were varied to manipulate acuity. Letter number might have a bigger effect in the lowacuity condition. Reaction time was the primary dependent variable.

\section{Method}

Subjects. The subjects were eight university students whose participation in the experiment satisfied an introductory course requirement. All subjects had normal or corrected-to-normal vision.

Apparatus and Stimuli. The stimulus letters were upright $\mathrm{T}$ 
and $\mathrm{L}$, and $\mathrm{T}$ tilted 30 deg clockwise from vertical. The letters were constructed with two equal-length straight lines subtending visual angles of $.78 \mathrm{deg}$ in length. The letters were drawn in black ink on a white background. In the high-acuity condition they were drawn with a Rapidograph No. 2 pen. The lines subtended $.047 \mathrm{deg}$ of visual angle in width. In the low-acuity condition the lines were drawn with a No. 000 pen and subtended a visual angle of $.023 \mathrm{deg}$. The letters were located $.78 \mathrm{deg}$ from the fixation point on the four corners of an imaginary square. The letter arrangement was the same as the foveal condition of Ambler and Finklea (1976). In the one-letter condition there was only one letter on the card. In the four-letter condition there were four letters, three of which were upright Ts on "Yes" trials. All four were upright Ts on "No" trials.

The stimuli were presented in a Gerbrands three-field tachistoscope at a viewing distance of $81 \mathrm{~cm}$. The luminances of the three fields were: $2.0 \mathrm{~cd} / \mathrm{m}^{2}$ (fixation), $3.9 \mathrm{~cd} / \mathrm{m}^{2}$ (stimulus), and $4.6 \mathrm{~cd} / \mathrm{m}^{2}$ (blank). The average room luminance was $2.2 \mathrm{~cd} / \mathrm{m}^{2}$. Reaction time was measured with a Hunter digital Klockounter which stopped when the subject pressed one of two microswitches.

Procedure. The experiment consisted of eight conditions arranged in a 2 by 2 by 2 design (two letter numbers, one vs. four, by two discrimination types, $\mathrm{L}$ vs. tilted $\mathrm{T}$, by two levels of acuity, high vs. low). The trials were blocked by letter number, discrimination type, and acuity. The subjects were run in two 24-trial blocks in each of the conditions, one block appearing on each of the 2 experimental days. In each block half of the stimulus presentations had a disparate figure (tilted $T$ or L) and the order of stimulus presentation was random. The letters occurred equally often in all four possible stimulus positions. Order of conditions was counterbalanced across days and across subjects.

Each experimental session started with 40 practice trials, 10 each in the four low-acuity conditions. On each trial subjects were instructed to press the appropriate response lever (Yes or No) as fast as possible, while keeping their error rate low (less than $5 \%$ ).

At the beginning of each trial, the fixation point was continually on. After giving a warning signal to the subject, the experimenter depressed a microswitch. The fixation point went off $500 \mathrm{msec}$ later and the stimulus field was presented for 100 msec. A 1-sec blank field followed the stimulus. Subjects were given feedback as to their speed and accuracy after each trial.

\section{Results}

The data were collapsed across Yes and No responses. The mean reaction times and errors were computed for each condition for all subjects. The means of these means are presented in Table 1. Errors were converted to percentages.

The reaction time data were analyzed with a 2 by 2 by 2 analysis of variance. The manipulation of acuity was significant $[F(1,7)=8.95, \mathrm{p}<.05, \mathrm{MSe}=315]$. There was no significant effect of letter type $[F(1,7)=$ $2.4, \mathrm{p}>.05, \mathrm{MSe}=789]$. There was a significant effect of number $[F(1,7)=15, p<.05, \mathrm{MSe}=301]$. It can be seen, however, that this was due to the T-L discrimination. Reaction time to this discrimination increased with letter number. There was essentially no effect of letter number on the $\mathrm{T}$-tilted $\mathrm{T}$ discrimination. This can be seen in the significant Number by Letter Type interaction $[F(1,7)=12.8, p<.05, \mathrm{MSe}=311]$. None of the other interactions was significant [Letter by Acuity,
Table 1

Mean Reaction Time (Milliseconds) and Percent Errors: Experiment 1

\begin{tabular}{lcccc}
\hline & \multicolumn{2}{c}{ One Letter } & \multicolumn{2}{c}{ Four Letters } \\
& L & Tilted T & L & Tilted T \\
\hline & \multicolumn{4}{c}{ High Acuity } \\
Reaction Time & 328 & 337 & 355 & 335 \\
Percent Error & 1.3 & 1.0 & 1.5 & 2.0 \\
& \multicolumn{4}{c}{ Low Acuity } \\
Reaction Time & 341 & 341 & 379 & 347 \\
Percent Error & 1.5 & 1.3 & 1.0 & 2.0 \\
\hline
\end{tabular}

$\mathrm{F}(1,7)=1.6, \mathrm{p}>.05, \mathrm{MSe}=285 ;$ Number by Acuity and Number by Letter by Acuity, $F<1]$.

A similar analysis was performed on the error data. There was no effect of acuity $(F<1)$. Nor was there an effect of letter number $[\mathrm{F}(1,7)=1.4, \mathrm{p}>.05, \mathrm{MSe}=1]$ or letter type $[\mathrm{F}(1,7)=1.3, \mathrm{p}>.05, \mathrm{MSe}=4]$. The Number by Letter Type interaction was not significant $[\mathrm{F}(1,7)=2.4, \mathrm{p}>.05, \mathrm{MSe}=63]$. There was a significant Number by Acuity interaction $[F(1,7)=9, p<.05$, MSe $=.06]$. However, number had a bigger effect on errors for the high- than for the low-acuity condition. Although there is no obvious explanation for this effect, it is clear that decreasing acuity did not increase the effect of letter number in this experiment. The Letter by Acuity and three-way interactions were not significant $(F<1)$.

\section{Discussion}

The most important result in this experiment is that a consistent effect of letter number on reaction time was obtained for the T-L discrimination. Little or no effect was obtained for the T-tilted $T$ discriminations. The fovea lacks the capacity to process even four letters in parallel when the discrimination involves the arrangement of one line relative to another within a figure. However, the evidence indicates that performance is little affected by number for line slant differences. This is consistent with previous results with four letters in the periphery and 18 letters in the fovea (Ambler \& Finklea, 1976).

There was no evidence that the Number by Letter interaction was increased by reducing acuity. However, there was a small but significant Number by Acuity interaction in the error data. The difference between one and four letters was larger for the highthan for the low-acuity condition. However, further inspection of the data showed that this difference was mainly due to the T-L discrimination. For this discrimination there were more errors in the four- than in the one-letter discrimination in the high-acuity condition. However, this pattern reversed for the low-acuity targets. Is this reversal reliable? We ran another experiment identical to the low-acuity condition of the present experiment. The reaction time data showed a pattern consistent with that of the present experiment (T-L: one letter $=345 \mathrm{msec}$, four letters $=369 \mathrm{msec}$; $\mathrm{T}$-tilted $\mathrm{T}$ : one letter $=344 \mathrm{~m} \mathrm{sec}$, four letters $=320 \mathrm{msec}$ ). However, the error data did not replicate the pattern shown in the low-acuity condition of Experiment 1 (T-L: one letter $=2.8 \%$, four letters $=3.6 \%$; T-tilted $\mathrm{T}:$ one letter $=1.6 \%$, four letters $=2.3 \%$ ).

The consistent finding across our experiments is that letter number affects the T-L discrimination. Our expectation that such a distributed attention effect might be magnified by a degraded-acuity condition was clearly not confirmed. 


\section{EXPERIMENT 2}

Experiment 1 showed that even for foveal processing there is not enough capacity to consider four figures simultaneously for a T-L discrimination. However, to process these figures the subject's attentional field would have to cover the entire 1-deg area that is considered foveal. Perhaps parallel processing could be achieved if the letters were separated by a smaller distance. In Experiment 2 we attempted to replicate the findings of Experiment 1 to see if the results would be influenced by the area of stimulus display.

\section{Method}

Subjects. The subjects were eight university students, with normal or corrected-to-normal vision. Their participation in the experiment satisfied an introductory course requirement.

Apparatus and Stimuli. The apparatus was the same as that used in Experiment 1. The stimuli were created in the same way with two exceptions. First, the stimuli were located in a diamond pattern around the fixation point. That is, there was a letter position directly above and below the fixation point There were two more letter positions directly to the left and right of the fixation point. Second, the stimuli were varied in their size and distance from the fixation point. In the large condition the letters were composed of lines $.57 \mathrm{deg}$ in visual angle, with their midpoints located 1.16 deg from the fixation point. The medium condition had letters made from .28-deg lines located $.57 \mathrm{deg}$ from fixation. Letters made up of .14-deg lines and located $.28 \mathrm{deg}$ from fixation made up the small condition. For all letters, line wid th was $.047 \mathrm{deg}$.

Procedure. With the following exceptions, the procedure was the same as that used in Experiment 1.

The experiment consisted of 12 conditions in a 2 by 2 by 3 design. There were two letter types (tilted $T$ and $L$ ), two letter numbers (one and four), and three letter sizes (large, medium, and small). The experiment was run in three sessions. The first session lasted $1.5 \mathrm{~h}$ and the last two were $1 \mathrm{~h}$ each. Session 1 started with four practice blocks of 10 trials each in the four large-letter conditions. Each condition run that day was preceded by five warmup trials. The trials were blocked by conditions. On each day each condition was run for one block of 16 trials each. One-half of the randomly arranged trials required a Yes response. Disparate figures occurred equally often in the four-letter positions. The order of conditions was counterbalanced across days and subjects.

\section{Results}

As in Experiment 1, the data from Yes and No responses were combined. Mean reaction time and percent errors are presented in Table 2.

The reaction time data was analyzed with an analysis of variance. The smaller display size produced slower reaction times $[\mathrm{F}(2,14)=56, \mathrm{p}<.05, \mathrm{MSe}=200]$. There was no main effect for letter type $[F(1,7)=1.15$, $\mathrm{p}>.05, \mathrm{MSe}=115]$. There was an effect of letter number $[F(1,7)=67, p<.05, \mathrm{MSe}=166]$. However, it can be seen that this effect was due to the T-L discrimination. This conclusion is supported by the Letter Type by Number interaction $[\mathrm{F}(1,7)=40, \mathrm{p}<.05$, MSe $=$ 218]. There was no Letter Type by Size interaction $[F(2,14)=1.4, p>.05$, MSe $=110]$. The Number by Size and three-way interactions produced $\mathrm{F}<1$.
Table 2

Mean Reaction Time (Milliseconds) and Percent Errors: Experiment 2

\begin{tabular}{llcccr}
\hline Display & & \multicolumn{2}{c}{ One Letter } & \multicolumn{2}{c}{ Four Letters } \\
Size & & Tilted T & L & Tilted T & L \\
\hline \multirow{2}{*}{ Large } & RT & 373 & 359 & 375 & 399 \\
& Error & 3.4 & 2.9 & 1.3 & 3.4 \\
\multirow{4}{*}{ Medium } & RT & 378 & 365 & 382 & 404 \\
& Error & 3.1 & 2.9 & 1.3 & 2.6 \\
\multirow{3}{*}{ Small } & RT & 411 & 389 & 414 & 431 \\
& Error & 4.9 & 2.6 & 2.1 & 3.6 \\
\hline
\end{tabular}

A similar analysis was performed on the error data. The main effects for letter type, Letter Type by Size interaction, and Number by Size interaction all resulted in $F<1$. The size main effect was not significant $[F(2,14)=2, p<.05, \mathrm{MSe}=68]$, nor was the three-way interaction $[\mathrm{F}(2,14)=1.5, \mathrm{p}>.05, \mathrm{MSe}=43]$.

The effect of letter number, however, approached significance $[F(1,7)=5.4, p<.10, \mathrm{MSe}=.86]$. It can be seen that this is due to the tilted-T discrimination, which was easier in the four-letter than in the oneletter condition. There was also a Letter Type by Number interaction $[\mathrm{F}(1,7)=18, \mathrm{p}<.05, \mathrm{MSe}=.56]$. The interaction for error rate showed the same thing as the interaction for reaction time. When shifting from one to four letters, the tilted-T discrimination became easier, but the $\mathrm{L}$ discrimination became more difficult.

\section{Discussion}

Previously, Amber and Finklea (1976) failed to find a statistically reliable effect of letter number on discrimination when comparing one and four letters. However, in both of the present experiments we found a significant number effect for the T-L discrimination but not for the $\mathrm{T}$-tilted $\mathrm{T}$ discrimination using a reaction time measure. When attention is spread out over four letters, the T-L discrimination becomes more difficult. In Experiment 2, this effect was not influenced by a manipulation of the retinal area covered by the letters. Even for the smallest array, number influenced T-L discrimination.

\section{GENERAL DISCUSSION}

The data continue to support the conclusion that T-tilted T discrimination is essentially a parallel process. It is possible it is made with a very fast sequential process that our measurements cannot detect. However, the results of Experiment 2, in which there was a trend toward more accurate performance for this discrimination in the four- than in the one-letter condition, suggests that this is not the case.

One explanation for the degraded performance in the T-L discrimination with four letters is that it is due to lateral masking. The results of Experiment 2 are inconsistent with such an explanation. Bringing the letters closer together should increase the magnitude of a masking effect. This manipulation did not increase the magnitude of the one- vs. four-letter difference for the $\mathrm{T}-\mathrm{L}$ discrimination.

The result may also be explained in terms of the limited capacity of an attention mechanism (Beck \& Ambler, 1973). Within the framework of this explanation, the results indicate that the capacity of the mechanism in the fovea is not increased by reducing the area covered by the array.

The effect of these basic discriminability differences for multielement arrays can be seen in the "grouping" phenomena 
(Beck, 1974) and may be involved in solving the visual system's problems of form segregation and integration.

\section{REFERENCE NOTE}

1. Prinzmetal, W., \& Banks, W. Simultaneous vs. successive presentation of visual arrays revisited. Paper presented at the annual meeting of the Psychonomic Society, Washington, D.C., 1977.

\section{REFERENCES}

Ambler, B., \& FinkleA, D. The influence of selective attention in peripheral and foveal vision. Perception \& Psychophysics, 1976, 19, 518-524.

BECK, J. Relation between similarity grouping and peripheral discriminability. Journal of Experimental Psychology, 1974, 102, 1145-1147.
Beck, J., \& Ambler, B. The effects of concentrated and distributed attention on peripheral acuity. Perception \& Psychophysics, 1973, 14, 225-230.

KerEN, G., \& Skelton, J. On selecting between theories of selective attention. Perception \& Psychophysics, 1976, 20, 85-86. Shiffrin, R. M. Attentional control. Perception \& Psychophysics, 1977, 21, 93-94.

Shiffrin, R. M., \& Gardner, G. T. Visual processing capacity and attentional control. Journal of Experimental Psychology, 1972, 93, 72-82.

Shiffrin, R. M., McKay, D. P., \& Shaffer, W. O. Attending to forty-nine spatial positions at once. Journal of Experimental Psychology: Human Perception and Performance, 1976, 2, 14-22.

(Received for publication February 2, 1978.) 